\section{BRCA1 mutation carriers}

BRCA1 mutation carriers are at increased risk for both breast and ovarian cancers. The Consortium of Investigators of Modifiers of BRCA1/2 (CIMBA) have previously reported a two-stage genomewide association study (GWAS) for modifiers of breast or ovarian cancer risk in BRCA1 mutation carriers (Nat. Genet. 42, 885-892, 2010), identifying a 19p13 locus associated with breast cancer risk for individuals with $B R C A 1$ mutation. Fergus Couch and colleagues now report a large-scale replication study including $11,705 B R C A 1$ mutation carriers from 45 centers in 25 countries (PLoS Genet. 9, e1003212, 2013). These samples were all genotyped using the custom iCOGS array, which included 32,557 SNPs selected from the original GWAS. The authors identified a new susceptibility locus at 1q32, containing the MDM4 gene, associated with breast cancer risk for $B R C A 1$ mutation carriers and also identified two new loci associated with ovarian cancer risk for BRCA1 mutation carriers at $17 q 21.31$ and $4 q 32.2$, with the latter representing the first locus to modify cancer risk specifically in BRCA1 mutation carriers. The authors also replicated previous associations and report a total of ten and seven loci associated, respectively, with breast and ovarian cancer risk in BRCA1 mutation carriers. On the basis of combinedSNP profiles, they report large differences in the predicted risk of developing breast or ovarian cancer for the $5 \%$ of BRCA1 mutation carriers at highest and lowest risk.

\section{BRCA2 mutation carriers}

A previous study from the Consortium of Investigators of Modifiers of $B R C A 1 / 2$ (CIMBA) reported a genome-wide association study (GWAS) for breast cancer risk in BRCA2 mutation carriers (PLoS Genet. 6, e1001183, 2010), identifying only known loci previously associated with breast cancer risk in the general population. Kenneth Offit and colleagues now report an extended replication, including genotyping using the iCOGS array, of 3,881 BRCA2 mutation carriers with breast cancer and 4,330 without breast cancer from CIMBA (PLoS Genet. 9, e1003173, 2013). They selected 19,029 SNPs from the initial GWAS for inclusion on the iCOGS array. The authors replicated previous breast cancer susceptibility loci and also identified a new susceptibility locus at 6p24 associated with breast cancer risk for BRCA2 mutation carriers but not with breast cancer for $B R C A 1$ mutation carriers or the general population. This represents the first $B R C A 2$-specific breast cancer association. Using a data set from The Cancer Genome Atlas (TGCA), they found that rs9348512 was associated with increased expression of the nearby gene GCNT2 in breast tumors. The authors used a combinedSNP risk profile of 14 SNPs known to modify risk in BRCA2 mutation carriers, predicting $21-47 \%$ risk of developing breast cancer by the age of 80 years for the $5 \%$ of the BRCA2 mutation carriers at lowest risk compared to $83-100 \%$ risk for the $5 \%$ at highest risk.

\section{Breast cancer associations in east Asians}

To extend findings of breast cancer association studies that have primarily been conducted in European-ancestry populations, Wei Zheng and colleagues systematically examined the association of known breast cancer susceptibility loci in east Asian women (Hum. Mol. Genet. doi:10.1093/hmg/ddt089, 27 March 2013). They genotyped 70 SNPs at

Research Highlights written by Orli Bahcall.
67 known susceptibility loci in 23,637 breast cancer cases and 25,579 controls of east Asian ancestry from the Asia Breast Cancer Consortium (ABCC) as well as Asian samples from COGS. They found that variants at 31 of these loci showed nominal association with breast cancer risk and that 21 of these met a Bonferroni-corrected significance level. This study offers the most comprehensive analysis of breast cancer risk variants in east Asians so far, but further studies and fine mapping of these regions in both European and Asian populations are needed to characterize population-specific differences in breast cancer susceptibility.

\section{Fine mapping at the $11 q 13$ locus}

Alison Dunning and colleagues report fine mapping of the $11 \mathrm{q} 13$ breast cancer susceptibility locus (Am. J. Hum. Genet. doi:10.1016/j. ajhg.2013.01.002, 27 March 2013). The original genome-wide association study (GWAS)-identified SNP tags a linkage disequilibrium block that spans $683 \mathrm{~kb}$, and the authors selected 731 SNPs from this region to include on the iCOGS array. They genotyped 89,050 individuals of European ancestry and 12,893 individuals of Asian ancestry, all from studies included in BCAC. They identified 204 SNPs associated with overall breast cancer risk, finding that these were all associated with estrogen receptor (ER)-positive but not ER-negative breast cancer. Using stepwise logistic regression, they identified three independently associated SNPs. They selected five promising candidate SNPs for functional studies but did not detect any significant association of these SNPs with the expression of local genes in normal breast tissue or tumor samples. Using chromatin immunoprecipitation with sequencing (ChIP-seq) data from MCF7 cells, they found that these SNPs fell within two putative regulatory elements. Chromatin interaction analysis by paired-end tag sequencing (ChIA-PET) and chromosome conformation capture (3C) analyses showed long-range interactions between these regulatory elements and the CCND1 promoter and/or terminator. Further functional studies identified a candidate causal variant in the putative CCND1 enhancer, which affected the binding of the ELK4 transcription factor. A second candidate causal variant, located within a silencer element that physically interacts with the CCND1 enhancer, affected binding of the GATA3 transcription factor.

\section{Fine mapping at the TERT locus}

Zsofia Kote-Jarai and colleagues report fine mapping of associations to prostate cancer susceptibility at the TERT locus using high-resolution genotyping and imputation (Hum. Mol. Genet. doi:10.1093/hmg/ddt086, 27 March 2013). The authors genotyped 134 SNPs across the TERT locus using the custom iCOGS array or Sequenom MassArray iPlex in 22,301 cases and 22,320 matched controls from 23 studies included in the PRACTICAL Consortium. They initially genotyped 114 SNPS across $135 \mathrm{~kb}$ of the SLC6A18-TERT-CLPTM1L region and then narrowed their focus to a 20-kb interval that included variants with stronger association. They further tested association for an imputed set of 1,094 SNPs. They identified 44 SNPs associated with prostate cancer risk at $P<1 \times 10^{-5}$. With stepwise logistic regression, they were able to identify four SNPs showing independent association, suggesting four separate regions influencing susceptibility to prostate cancer. They examined gene expression of TERT and CLPTM1L in 195 normal (histologically benign) prostate tissue samples isolated from men with elevated prostate-specific antigen (PSA) levels. They found protective alleles of four SNPs in one region associated with higher expression of TERT. 